\title{
Tecnura
}

\section{Nanomateriales celulósicos para la adsorción de contaminantes emergentes}

\section{Cellulosic nanomaterials for adsorption of emerging contaminants}

\author{
Belkis Coromoto Sulbarán Rangel ${ }^{1}{ }^{(\mathbb{D}}$ Abigail Eloísa Madrigal Olveira² ${ }^{\mathbb{D}}$, Víctor Hugo Romero \\ Arellano $^{3}$ y Carlos Alberto Guzmán González ${ }^{4}$
}

Fecha de recepción: 13 de mayo de 2019

Fecha de aceptación: 23 de agosto de 2019

Como citar: Sulbarán, B.C., Madrigal, A.E., Romero, V.H. y Guzmán, C.A. (2019). Nanomateriales celulósicos para la adsorción de contaminantes emergentes. Tecnura, 23(62), 13-20. https://doi.org/10.14483/22487638.15451

\section{Resumen}

Contexto: En la actualidad la nanotecnología posee múltiples áreas de acción que, debido a su naturaleza, la misma puede instrumentarse con amplia versatilidad, dado que, gran cantidad de avances en nanotecnología basan sus estudios en cómo optimizar procesos cotidianos e industriales y en cómo favorecer al medio ambiente. Aunado a ello, la manipulación de la materia a este nivel permite crear soluciones con mayor proyección en impacto científico, social y económico. Para fines de esta investigación, se mostrarán resultados a nivel de laboratorio usando nanomateriales celulósicos para la adsorción de contaminantes emergentes tipo antibióticos. Método: Esta investigación se realizó a nivel de laboratorio, en donde se modificó por métodos químicos celulosa para obtener nanocelulosa por oxidación. Se realizó una caracterización de material obtenido por técnicas de espectroscopia y se evaluó la adsorción de contaminantes emergentes tipo antibiótico como la ciprofloxacina.

Resultados: Los nanomateriales celulósicos tienen potencial para ser usados en tratamiento de agua terciario en la eliminación de contaminantes emergentes como la ciprofloxacina. Los resultados muestran que el nanomaterial celulósico adsorbe la ciprofloxacina en un $27 \%$.

Conclusiones: Las membranas de nanocelulosa tienen potencial para ser usadas en un sistema de purificación de agua; aquellas echas solo con celulosa presentaron un menor porcentaje de adsorción del contaminante que las membranas con nanocelulosa.

Palabras clave: nanomateriales, adsorción, nanotecnología, contaminantes emergentes.

\section{Abstract}

Context: At present, nanotechnology has multiple areas of action that, due to its nature, can be

1 Ingeniera Forestal, maestra en Ciencias de Productos Forestales, doctora en Ciencias de Materiales. Profesora investigadora asociada B al Centro Universitario de Tonalá, Universidad de Guadalajara. Guadalajara, México. Contacto: belkis.sulbaran@academicos.udg.mx

2 Ingeniera en Nanotecnología. División de Ingenierías e Innovación Tecnológica. Centro Universitario de Tonalá, Universidad de Guadalajara. Guadalajara, México. Contacto: shapys_abigail@hotmail.com

3 Licenciado en Física, magister en Ciencias, doctor en Ciencias. Profesor investigador titular. Centro Universitario de Tonalá, Universidad de Guadalajara. Guadalajara, México. Contacto: victor.romero@cutonala.udg.mx

4 Licenciado en Química, magister en Ciencias, doctor en Ciencias, posdoctor de la Universidad de California Campus Riverside. Profesor investigador asociado B. Centro Universitario de Tonalá, Universidad de Guadalajara. Guadalajara, México. Contacto: carlos.guzman@ cutonala.udg.mx 
implemented with great versatility, given that a large number of advances in nanotechnology base their studies on how to optimize every day and industrial processes and how to favor the environment ambient. In addition to this, the manipulation of matter at this level allows creating solutions with greater projection in scientific, social and economic impact, for the purpose of this research, results will be shown at the laboratory level using cellulosic nanomaterials for the adsorption of emerging antibiotic contaminants.

Method: This research was carried out at the laboratory level where it was modified by chemical cellulose methods to obtain nanocellulose by oxidation with TEMPO. A characterization of material obtained by spectroscopy techniques was performed and the adsorption of emerging antibiotic contaminants such as ciprofloxacin was evaluated.

Results: Cellulosic nanomaterials have the potential to be used in tertiary water treatment in the removal of emerging contaminants such as ciprofloxacin. The results show that the cellulosic nanomaterial adsorbs ciprofloxacin by $27 \%$.

Conclusions: The nanocellulose membranes have the potential to be used in a water purification system, those membranes made only with cellulose had a lower percentage of adsorption of the contaminant than the membranes with nanocellulose.

Keywords: nanomaterials, adsorption, nanotechnology, emerging pollutants.

\section{INTRODUCCIÓN}

El agua es uno de los recursos más abundantes y valiosos para la humanidad, pero su disponibilidad para el consumo humano es mínima. "Los eventos climáticos extremos ocasionan grandes retos para la sostenibilidad social, económica y medioambiental" (González, Carvajal y Loiza, 2016, p. 101). En este sentido, siendo la cantidad tan escaza, existen datos que confirman que más de 700 millones de personas no tuvieron la provisión de agua potable y lo que puede ser un poco más preocupante es donde el agua sí está disponible los costos han incrementado debido al aumento del costo de la energía, al igual que la sobrepoblación, incluso otros problemas ambientales que puedan afectarlo (Scholz, 2016).

A pesar de los problemas mencionados, también se busca la manera de solucionar los escases de agua potable, disminuir la contaminación misma del valioso líquido, por medio del uso de diseños de nanomateriales. Se han desarrollado diferentes investigaciones para reducir el impacto de la contaminación en el agua potable, pero no solo es la remediación del agua, sino que también el costo debe ser asequible (Gautam y Chattopadhyaya, 2016). "En los últimos años, muchos países, incluyendo Colombia, han desarrollado legislación de tipo ambiental que limitan los vertimientos que habían venido deteriorando a través del tiempo sus recursos hídricos" (Suarez, García y Vaca, 2012, p. 186).

"México por su lado, padece una serie de problemas ambientales que comprometen la sostenibilidad de su desarrollo" (González, Retamoza, Álbores y Guerrero, 2016, p. 92). Por ello, tratar el agua sirve para intentar alcanzar su potabilización, para lo cual se recurre al acondicionamiento y uso de tecnologías que modifican y eliminan contaminantes como patógenos e impurezas no deseadas, que hacen que el agua no sea apta para consumo, tomando en cuenta las normas vigentes sobre niveles máximos permitidos. Los límites permisibles varían conforme al país donde se aplique el tratamiento y va de acuerdo con el tipo de contaminantes, ya que, si estos últimos están presentes en el agua y en límites mayores a los señalados, el agua se debe tratar. Se recomienda el tratamiento o modificación del agua para que cumpla con el nivel máximo de contaminantes (Olvera, Silva, Robles-Belmont y Lau, 2017; Scholz, 2016). El agua de Iluvia también podría ser una fuente de aprovechamiento del recurso, sin embargo, no se ha tenido en cuenta debido a que se necesita profundizar en estudios sobre costos 
y beneficios para su recolección y aprovechamiento (León, Córdoba y Carreño, 2016).

Algunos de los procesos de tratamiento más comunes para aguas superficiales y subterráneas son procesos físicos, químicos o mecánicos que remueve contaminantes y modifican algunas de las características del agua antes de otros procesos adicionales. En ocasiones, la adición de sustancias químicas para alterar la calidad del agua es la única técnica de tratamiento usada (Scholz, 2016).

La nanotecnología ofrece alternativas, ya que algunos nanomateriales son vertidos en cuerpos de agua en aras de tratar con mayor eficacia, pues capturan residuos químicos u orgánicos $(\mathrm{Qu}$, Alvarez y Li, 2013). Otras cumplen la función de acelerar procesos como adsorción y catálisis; algunos más, inclusive, prometen detectar el grado de contaminación y, en función de eso, determinar el método a seguir, esto cuando los residuos químicos son de gran magnitud y la velocidad a la que se hace es tal, que los métodos tradicionales son ya ineficientes. Por ello, con las nuevas formas del tratamiento del agua se busca, desde el principio precautorio, establecer parámetros de riesgo para el uso de nanomateriales con este propósito (Adeleye et al., 2016). Hoy, hay una tendencia hacia los métodos de remediación, especialmente aqueIlos que investigan nanomateriales para filtración y adsorción. Pese a ello, la aplicación de estas tecnologías para combatir la problemática del agua continú, mientras que el vital líquido es escaso y solo un porcentaje menor al $30 \%$ pasa por un tratamiento adecuado (Olvera et al., 2017).

En esta investigación se presentan los resultados del uso de un nanomaterial obtenido de polímeros biodegradables como la celulosa. Se muestran la adsorción de contaminantes emergentes tipo antibióticos, como la ciprofloxacina.

\section{METODOLOGÍA}

Se utilizó celulosa del bagazo de agave, la cual fue modificadaa escala nanocon procesosquímicoscon oxidación con N-oxil-2,2,6,6-tetrametilpiperidina
(TEMPO), siguiendo la metodología propuesta por Lin, Bruzzese y Dufresne (2012). El proceso de oxidación ocurre al agregar $9 \mathrm{ml}$ de $\mathrm{NaClO}$ (10$13 \%$ wt) a temperatura ambiente con agitación de 500 rpm durante tres horas. La reacción se finaliza agregando $5 \mathrm{ml}$ de etanol anhídrido. Luego la solución se coloca en diálisis por dos días.

Se calcula el grado de oxidación (degree of oxidation-DO) y se expresa como la relación entre la cantidad de grupos hidroximetilo oxidados y los grupos hidroximetilo totales, que se determinó mediante valoración conductimétrica. Se suspende una muestra de $50 \mathrm{mg}$ de nanocelulosa oxidad con TEMPO en $15 \mathrm{ml}$ de soluciones de ácido clorhídrico 0,01 mol/L. Después de 10 minutos de agitación, la suspensión se titula con 0,005 mol/L de solución de $\mathrm{NaOH}$ bajo presión. La conductividad se controla usando un medidor de conductividad durante todo el proceso de titulación. La valoración se termina cuando el pH alcanza 11. El DO se calcula mediante la ecuación (1).

$$
\text { DO }=\frac{162 \times C \times\left(V_{2}-V_{1}\right)}{m-36 \times C \times\left(V_{2}-V_{1}\right)} \times 100 \%
$$

Donde $C$ es la concentración de $\mathrm{NaOH}, V_{1}$ y $V_{2}$ son el volumen de $\mathrm{NaOH}$, y $m$ es el peso de la muestra secada al horno.

\section{Preparación de las membranas nanoestructuradas}

Se elaboraron membranas a partir de soluciones de nanocelulosa TEMPO y celulosa de agave, las cuales se usarán directamente en los procesos de adsorción de contaminantes. Las membranas se elaboraron por casting. Este método se caracteriza por ser muy simple; en principio se prepara la solución y se vierte en una caja de Petri. El solvente se elige de manera que se evapore fácilmente. Las variables posibles de controlar son la concentración, el solvente y en ocasiones se utiliza ultrasonido o magnetismo para intentar controlar la dirección predominante de las fibras (Abdul Khalil et al., 2014). La celulosa y la nanocelulosa 
obtenidas del bagazo de agave fueron caracterizadas por técnicas analíticas y de espectroscopia. El objetivo fue establecer una correlación entre la estructura, forma y composición química de los polímeros obtenidos con sus propiedades. Las técnicas de caracterización fueron: espectroscopia infrarroja, difracción de rayos $X$, microscopia electrónica de barrido y microscopia de fuerza atómica. Adicionalmente, se evaluó la porosidad de la membrana midiendo los poros en las fotografías de microscopia con ayuda de programa ImageJ.

\section{Prueba de adsorción de contaminantes}

Para determinar el potencial de uso de las membranas en un sistema de purificación de agua en esta investigación se preparó un modelo simplificado de agua sintética utilizando agua destilada y un antibiótico comercial Ilamado ciprofloxacina en proporción de 1l de agua destilada por $30 \mathrm{~g}$ de ciproflaxacina (Garcia-Alonso et al., 2018). Esta es un contaminante emergente que, según reportes, se encuentra en aguas para consumo. Utilizando espectroscopia UV-Vis se analizó el agua sintética preparada con ciproflaxacina, se realizó una curva de calibración de la solución de ciproflaxacina. La curva de calibrado se construyó midiendo la señal analítica de absorbancia en cada una de las disoluciones de ciproflaxacina de $1 \%$ al $50 \%$. En el eje de ordenadas se asignó el valor de la señal medida y en el eje de abscisas, la concentración de la disolución. Las variables que se consideraron en el proceso fueron: tiempo, concentración y velocidad de agitación. Se hicieron medidas de UV-vis del agua a una hora para determinar si era adsorbido el contaminante. En este caso, el porcentaje de adsorción o la eficiencia de eliminación (\%) se calculó de acuerdo con la ecuación (2).

Eficiencia de la adsorción $=[(\mathrm{Ci}-\mathrm{Ct}) / \mathrm{Ci}] \times 100$

Donde Ci y Ct son las concentraciones iniciales y en cualquier momento $t$ (horas), respectivamente, expresadas en $\mathrm{mg} / \mathrm{L}$ en CIP, empleando una espectroscopia UV-Vis. Todo el experimento se llevó a cabo por triplicado, y los datos que se muestran son promedios con cálculos de desviación estándar, usando Statistics and Machine Learning Toolbox con MatLab versión 2015.

\section{RESULTADOS}

En la figura 1, se observa la caracterización del nanomaterial por espectroscopia infrarroja en donde la nanocelulosa TEMPO, a diferencia de la celulosa de agave, presenta una nueva banda a 1600 $\mathrm{cm}^{-1}$, que corresponde a la vibración de estiramiento $\mathrm{C}=\mathrm{O}$ de los grupos carboxilo en su forma ácida. Esto sugiere que los grupos hidroximetilo de la unidad de D-glucosa se convirtieron en grupos carboxilo con éxito.

Para el análisis de la morfología de la celulosa y nanocelulosa, las muestras fueron analizadas en el microscopio electrónico de barrido a diferentes magnificaciones con el propósito de observar el cambio entre la celulosa y la nanocelulosa TEMPO. En la figura 2 se presenta la imagen SEM de la celulosa de agave sin la modificación TEMPO observando fibras con un grosor que varía desde 10-40 $\mu \mathrm{m}$.

Con las muestras de nanocelulosa oxidadas con TEMPO en las condiciones mencionadas anteriormente, se prepararon membranas (figura 3). En cuanto a la morfología de las muestras a escala macroscópica, se observan como una lámina de pulpa celulósica generalmente de conformación irregular. Para analizar mejor la morfología de las nanofibras de celulosa, las muestras fueron observadas en el microscopio electrónico de barrido a diferentes magnificaciones, con el propósito de ver la conformación de las membranas y porosidad.

Las membranas fueron colocadas en una solución de agua sintética con $30 \mathrm{ml} / \mathrm{L}$ de concentración de la ciproflaxacina por una hora. Se hicieron mediciones por espectroscopia UV y a partir de la curva de calibración fue determinado el porcentaje de remoción del contaminante por las membranas (tabla 1). 


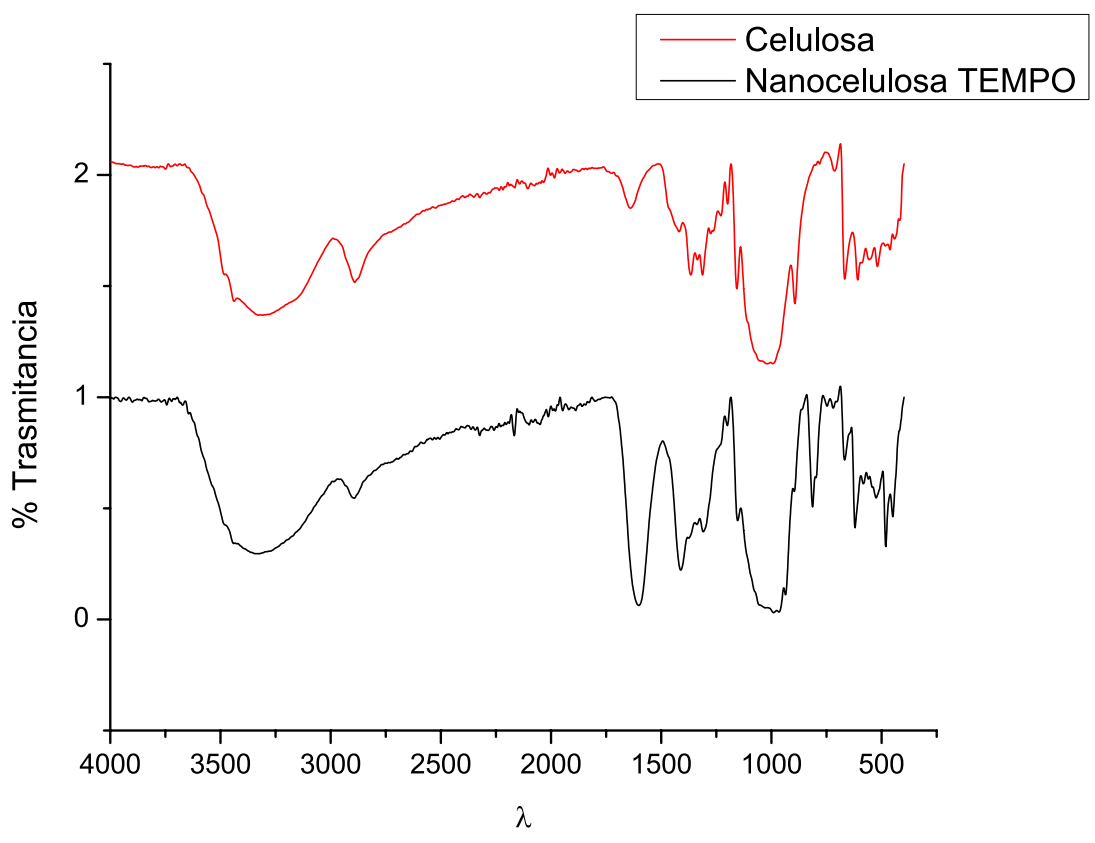

Figura. 1. Análisis de FTIR celulosa y nanocelulosa oxidada con TEMPO

Fuente: elaboración propia.
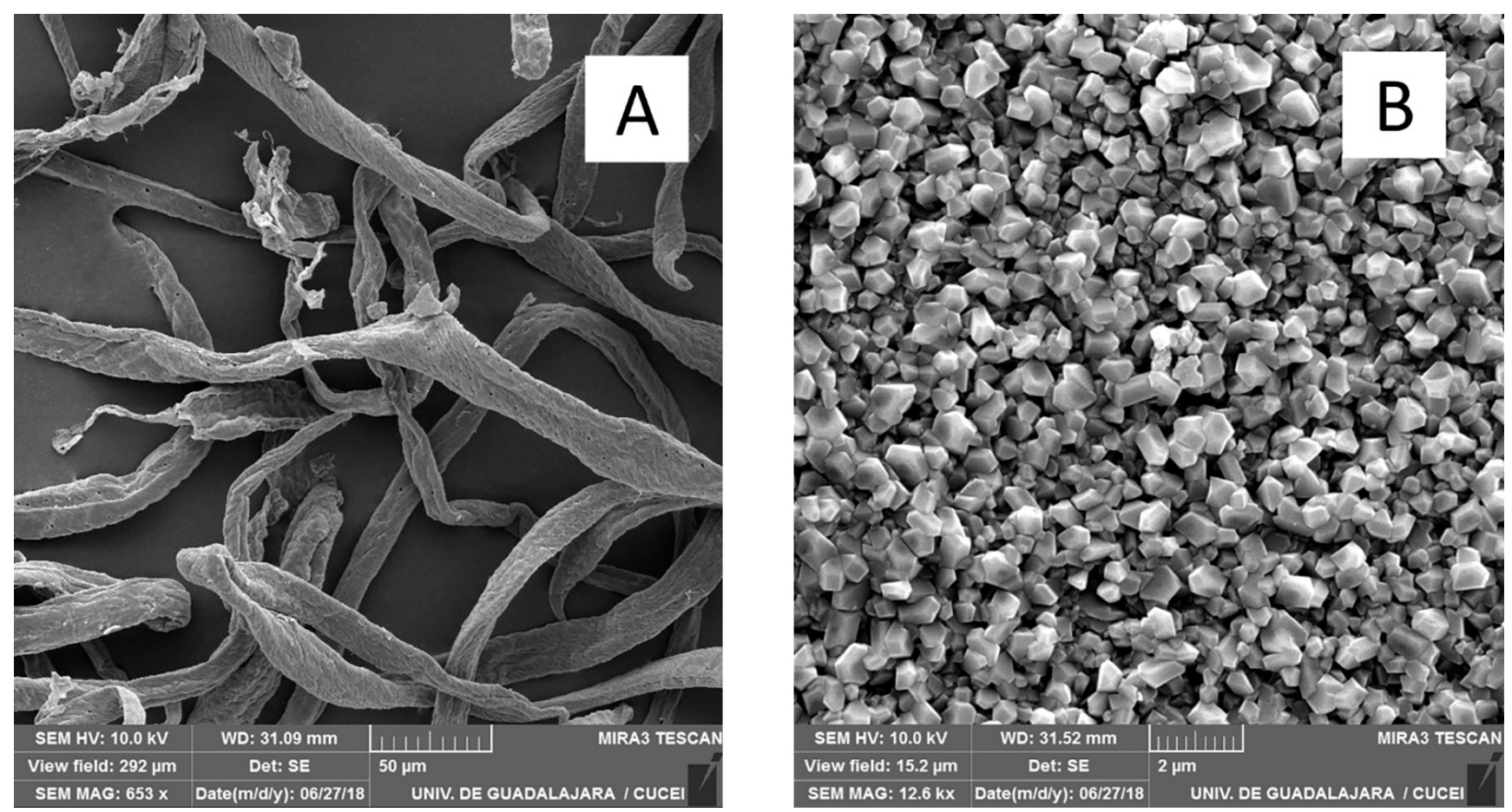

Figura 2. Imagen SEM. A. Celulosa de bagazo. B. Nanocelulosa oxidada con TEMPO

Fuente: elaboración propia. 


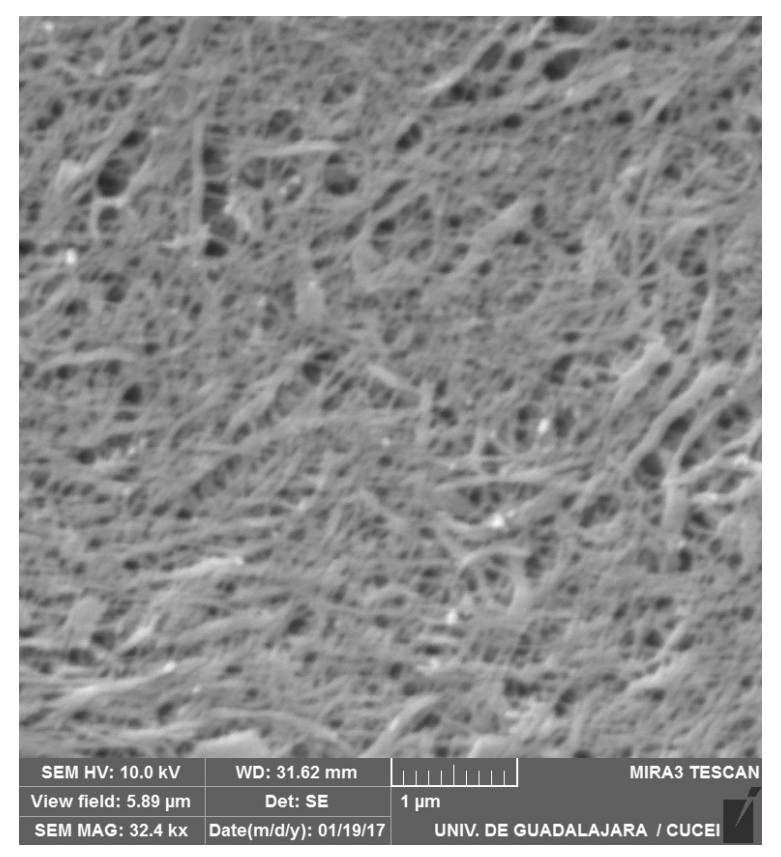

Figura 3. Imágenes SEM de la superficie de la membrana a escala microscópica $5 \mu \mathrm{m}$

Fuente: elaboración propia.

Tabla 1. Desempeño de las membranas de nanocelulosa TEMPO en la adsorción de ciploflaxacina

\begin{tabular}{cc}
\hline Membrana & \% de remoción \\
\hline $100 \%$ celulosa & $15,03 \pm 1.00$ \\
\hline $50 \%$ celulosa $50 \%$ nanocelulosa TEMPO & $27,76 \pm 6.01$ \\
\hline $70 \%$ celulosa $30 \%$ nanocelulosa TEMPO & $18,57 \pm 6.61$ \\
\hline
\end{tabular}

Fuente: elaboración propia.

La adsorción de la ciprofloxacina (CIP) por las membranas de nanocelulosa podría atribuirse a la atracción entre las superficies cargadas negativamente de la membrana y las cargas positivas de las moléculas CIP, similares a los reportados por otros autores y comparables al efecto del $\mathrm{pH}$ en la adsorción de CIP con minerales como las goethitas, esmectitas y caolín. Varios métodos de adsorción tienden a ser más efectivos en todos los ámbitos para remover la mayoría de contaminantes, orgánicos e inorgánicos. En general, se usan nanomateriales a base de carbono para atrapar las moléculas contaminantes dentro de las estructuras porosas; sin embargo, debido a que la adsorción remueve el contaminante (no lo elimina o lo transforma) se debe manejar de una manera adecuada ya que se tiene un peligroso desecho (Rostamian y Behnejad, 2018).

El intercambio de iones es otra forma de adsorción que se usa comúnmente para remover iones de metales pesados al igual que otros iones no metálicos; lo que sucede por este método es remplazar la solución con iones menos tóxicos, siendo este proceso muy característico en residuos de vapor donde existe una alta concentración de metales y otros iones (Thakkar, Wu, Wei y Mitra, 2015). 


\section{CONCLUSIÓN}

Las aplicaciones de la nanotecnología en la purificación del agua y remediación del ambiente se han considerado que tienen potencial. Con base en los resultados de la adsorción del contaminante emergente tipo ciprofloxacina, se concluye que los nanomateriales celulósicos podrían ser usados en un sistema terciario de tratamiento de agua, las membranas echas solo con celulosa presentaron un menor porcentaje de adsorción del contaminante que las membranas preparadas con nanocelulosa, siendo las membranas con $50 \%$ de nanocelulosa-TEMPO las que presentaron el porcentaje de adsorción más alto.

\section{REFERENCIAS}

Abdul Khalil, H.P.S., Davoudpour, Y., Islam, M.N., Mustapha, A., Sudesh, K., Dungani, R. y Jawaid, M. (2014). Production and modification of nanofibrillated cellulose using various mechanical processes: A review. Carbohydrate polymers, 99 (Supplement C), 649-665. https://doi.org/10.1016/j. carbpol.2013.08.069

Adeleye, A.S., Conway, J.R., Garner, K., Huang, Y., Su, Y. y Keller, A.A. (2016). Engineered nanomaterials for water treatment and remediation: Costs, benefits, and applicability. Chemical Engineering Journal, 286, 640-662. https://doi.org/10.1016/j. cej.2015.10.105

Garcia Alonso, J.A., Zurita Martinez, F., Guzmán González, C.A., Del Real Olvera, J. y Sulbarán Rangel, B.C. (2018). Nanostructured diatomite and its potential for the removal of an antibiotic from water. Bioinspired, Biomimetic and Nanobiomaterials, 7(3), 167-173. https://doi.org/10.1680/jbibn.18.00020

Gautam, R.K. y Chattopadhyaya, M.C. (2016). Chapter 1. Nanotechnology for Water Cleanup. En R.K. Gautam y M.C. Chattopadhyaya (eds.), Nanomaterials for Wastewater Remediation (1-18). Boston: Butterworth-Heinemann. https://doi.org/10.1016/ B978-0-12-804609-8.00001-7
González López, N., Carvajal Escobar, Y. y Loaiza Cerón, W. (2016). Análisis de sequías meteorológicas para la cuenca del río Dagua, Valle del Cauca, Colombia. Tecnura, 20(48), 101-113. https://10.14483/ udistrital.jour.tecnura.2016.2.a01

González, G., Retamoza, J.G., Álborez Arazate, H. y Guerrero de León, A. (2016). Gestión integral de cuencas hidrográficas: una alternativa a la sustentabilidad de los recursos hídricos en México. Lacandonia 10(1), 91-98. Recuperado de: https://cuid. unicach.mx/revistas/index.php/lacandonia/article/ down load/398/364/

León, A., Córdoba, J.C. y Carreño, U.F. (2016). Revisión del estado de arte en captación y aprovechamiento de agua lluvias en zonas urbanas y aeropuertos. Tecnura, 20(50), 141-153. https://10.14483/udistrital.jour.tecnura.2016.4.a10

Lin, N., Bruzzese, C. y Dufresne, A. (2012). TEMPO-Oxidized Nanocellulose Participating as Crosslinking Aid for Alginate-Based Sponges. ACS Applied Materials \& Interfaces, 4(9), 4948-4959. https://doi. org/10.1021/am301325r

Olvera, R.C., Silva, S.L., Robles-Belmont, E. y Lau, E.Z. (2017). Review of nanotechnology value chain for water treatment applications in Mexico. Resource-Efficient Technologies, 3(1), 1-11. https://doi.org/10.1016/j.reffit.2017.01.008

Qu, X., Alvarez, J. y Li, Q. (2013). Applications of nanotechnology in water and wastewater treatment. Water Research, 47(12), 3931-3946. https://doi.org/10.1016/j.watres.2012.09.058

Rostamian, R. y Behnejad, H. (2018). A comprehensive adsorption study and modeling of antibiotics as a pharmaceutical waste by graphene oxide nanosheets. Ecotoxicology and Environmental Safety, 147, 117-123. https://doi.org/10.1016/j. ecoenv.2017.08.019

Scholz, M. (2016). Chapter 2. Water Treatment. En M. Scholz (ed.), Wetlands for Water Pollution Control (pp. 9-11). 2a. ed. Ámsterdam: Elsevier. https://doi. org/10.1016/B978-0-444-63607-2.00002-2

Suárez, F., García, A. y Vaca, L. (2012). Identificación y evaluación de la contaminación del agua por 
curtiembres en el municipio de Villapinzón. Tecnura, 16(1), 185-194.

Thakkar, M., Wu, Z., Wei, L. y Mitra, S. (2015). Water defluoridation using a nanostructured diatom$\mathrm{ZrO} 2$ composite synthesized from algal Biomass. Journal of Colloid and Interface Science, 450, 239-

245. https://doi.org/10.1016/j.jcis.2015.03.017 